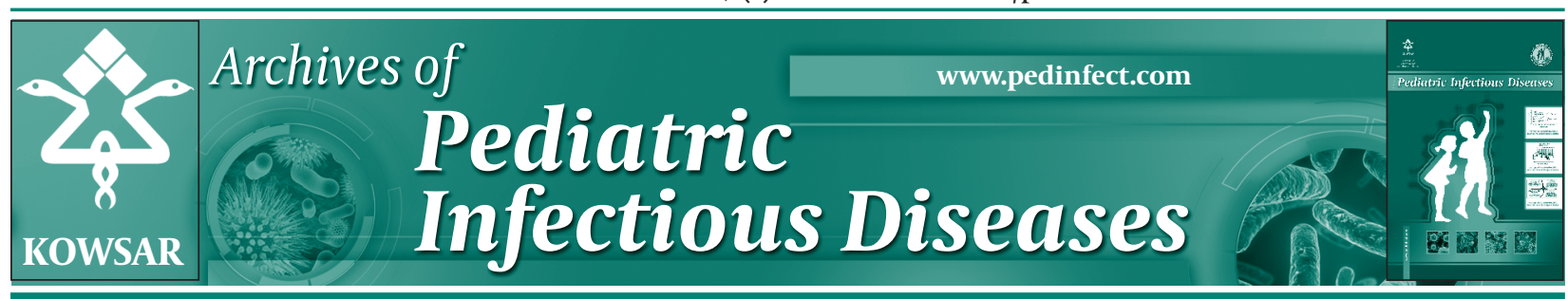

\title{
The Value of Renal Scintigraphy With DMSA for Assessing Vesicoureteral Reflux in Children With Suspected Urinary Tract Infection
}

\author{
Fariba Shirvani ${ }^{1 *}$, Mostafa Sharifian ${ }^{1}$, Masoumeh Mohkam ${ }^{1}$ \\ ${ }^{1}$ Pediatric Infections Research Center, Mofid Children Hospital, Shahid Beheshti University of Medical Sciences, Tehran, IR Iran
}

\begin{tabular}{l}
\hline A R T I C L E I N F O \\
\hline Article type: \\
Brief Report \\
\hline Article history: \\
Received: 05 Mar 2012 \\
Revised: 11 Jul 2012 \\
Accepted: 25 Jul 2012 \\
\hline
\end{tabular}

\begin{abstract}
A B S T R A C T
Background: Renal scintigraphy with technetium 99m labeled dimercaptosuccinic acid (99mTc-DMSA) is a traditional imaging technique commonly used to detect renal scar in patients with probable vesicoureteral reflux (VUR) and/or urinary tract infection (UTIs). We determined whether normal results of DMSA renal scan obviate the need for voiding cystourethrography (VCUG) in evaluating children with UTIs.

Materials and Methods: We observed medical records from June 2006 to April 2007 retrospectively of 208 children admitted with community acquired UTIs to Mofid children hospital (Tehran, IR/Iran) a teaching hospital in Tehran in which their age was between 2-120 months. The association between DMSA renal scan results and VCUG findings performed 48 hours and 1 month after the diagnosis was evaluated. To examine the accuracy of abnormal tive predictive value (NPV) and negative and positive likelihood ratio (LRs) were calculated. Results: VUR was seen in $14.0 \%$ of renal units with normal results of DMSA and $17.3 \%$ of renal units with abnormal DMSA findings. High grade VUR (grade III-V) was seen in 18 (7.1\%) of the abnormal findings of DMSA group and in (2.8\%) 1 of the normal DMSA results group $(P=0.56)$. In the group with previous UTI $(n=68)$, the sensitivity and NPV of abnormalities on DMSA renal scan for detecting the presence of VUR (grade III-V) were 100\%, and100\%, respectively. In the group without evidence of previous UTI, the sensitivity and NPV of abnormalities on DMSA renal scan for detecting the presence of VUR (grade III-V) were 93\% and 97\%, respectively. Totally the sensitivity and NPV of abnormalities on DMSA renal scan to detect the presence of VUR (grade III-V) were $94 \%$ and $97 \%$, respectively.

Conclusions: As a screening test, DMSA renal scan is a high sensitive technique to assess VUR (grade III-V) in children with UTI.
\end{abstract}

Urinary Tract Infections

Technetium Tc 99m Dimercaptosuccinic Acid DMSA results in predicting VUR, sensitivity, specificity, positive predictive value (PPV), negaVesico-Ureteral Reflux Child

Published by Kowsar Corp, 2012. cc 3.0.

Implication for health policy/practice/research/medical education:

Pyelonephritis is a common infection in children, and because of high association between pyelonephritis and Vesicoureteral Reflux, and difficulty in performing Voiding Cystourethrography in children, we decided to evaluate the rule of DimercatoSuccinic Acid in prediction of Vesicoureteral Reflux in pediatric Group.

- Please cite this paper as:

Shirvani F, Sharifian M, Mohkam M. The Value of Renal Scintigraphy With DMSA for Assessing Vesicoureteral Reflux in Children With Suspected Urinary Tract Infection. Arch Pediatr Infect Dis.2012;1(1): 27-30. DOI: 10.5812/pedinfect.4741

\footnotetext{
* Corresponding author: Fariba Shirvani, Pediatric Infections Research Center, Department of Pediatric Infectious Diseases, Mofid Children Hospital, Shariati

St., P.O.Box:15468-15514, Tehran, IR Iran. Tel:+98-2122859142, Fax:+98-2122226941, E-mail: fariba_shirvani@yahoo.com

DOI:10.5812/pedinfect.4741

(C) 2012 Pediatric Infections Research Center and Shahid Beheshti University of Medical Sciences; Published by Kowsar Corp.

This is an open access article distributed under the terms of the Creative Commons Attribution License (http://creativecommons.org/licenses/by/3.0), which permits unrestricted use, distribution, and reproduction in any medium, provided the original work is properly cited.
} 


\section{Background}

Significant numbers of young children develop a symptomatic, culture-confirmed urinary tract infection (UTIs) which may present with fever, cloudy urine, failure to thrive or nonspecific symptoms (1). Urine analysis has been acknowledged as a useful initial tool for investigation of UTIs. Urine culture, is necessary for the diagnosis of UTI in children whenever there is a clinical suspicion, or if urine dipstick testing results shows positive leukocyte esterase or nitrite activity (2).

In most affected children, vesicoureteral reflux (VUR) can be considered as the main underlying etiology and a serious risk factor for UTIs. In this context and due to frequent recommendations, routine imaging studies are used to improve the accuracy of diagnostic approach in children suggestive of VUR. A sensitive and specific traditional imaging technique commonly used to detect renal scars in children with UTI is renal scintigraphy with technetium99m labeled dimercaptosuccinic acid (99mTc-DMSA). Voiding cystourethrography (VCUG) identifies those with VUR, however neither VCUG nor DMSA is used specifically as a single diagnostic test to identify febrile UTI (3).

Although high efficacy of DMSA to confirm VUR has been proven in some studies, some other studies including infants with UTIs have shown that dilating VUR may occur when early DMSA scintigraphic results are normal. In fact, some previous studies have reported very low specificity and negative predictive values of DMSA in children with $\operatorname{VUR}(4,5)$, while some other reports confirmed increased negative predictive value of both-tests strategy (VCUG plus DMSA) (6).

Significant relationship exists between VUR (grades III V) and permanent renal damage (7) but Nondilating VUR (grades I-II) has low significance (8) and further follow up is not necessary in treatment protocol of these children with UTI (9). There remains considerable controversy over which way is the best to investigate children with UTI.

\section{Objectives}

The aim of the present study was to identify VUR among children with UTI by DMSA versus the gold standard of VCUG and examine the effectiveness of routine investigation by DMSA versus VCUG in infants with suspected UTIs.

\section{Materials and Methods}

We observed the medical records from June 2006 to April 2007 retrospectively of 208 children admitted with community-acquired UTI to Mofid children hospital (Tehran, IR Iran), a teaching hospital in Tehran. Inclusion criteria included: children with clinical symptoms compatible with UTI i.e. fever $>37.5^{\circ} \mathrm{C}$ (axillary) and any symptom of (dysuria, frequency, urgency, flank pain). Child was considered as UTI case if the following criteria were observed: clinical symptoms plus pyuria, (>10 WBC/HPF), or symptoms compatible with UTI Plus positive nitrite test, or symptoms plus pyuria plus one positive culture $>10^{5}$ colony $/ \mathrm{mm}^{3}$ with clean catch method (midstream or bag after genital wash with water and bag placement for 30 minutes in children $<3$ years old or positive pyuria plus bacteriuria plus one positive culture with one microorganism with $>10^{5}$ colony $/ \mathrm{mm}^{3}$ of urine with clean catch method. Children with recurrent UTIs with medical documentation such as medical record and previous positive urinalysis/culture results were also included. Exclusion criteria included: 1 ) Children with UTIs that were already hospitalized for other reasons and had normal urinalysis results at admission. 2) Those with manifestations or suggestive of obstructive uropathy on ultrasonograms. 3) Children with a chronic urinary disorder, such as neurogenic bladder, or a congenital disorder, including anatomic defects and meningomyelocele. Informed written consent was obtained; moreover they were not incurred for any extra charge. The following data was recorded: gender, age and weight at diagnosis, clinical manifestations, and blood and urine culture results. A patient's urine was cultured if abnormal results of dipstick, (positive leukocyte esterase or nitrite) or microscopy (pyuria $>10$ cells/high-power field) tests were found or if UTI was clinically suspected. All urine specimens for culture were collected using Clean Catch method and were immediately sent to culture. Children with proven infections underwent DMSA renal scan within 2 days and VCUG within 1 month after acute infection. Technetium 99m labeled dimercaptosuccinic acid (99mTc-DMSA). Renal scanning was performed according to a standard protocol (10). A positive DMSA result was defined as scarring; focal or diffuse areas of reduced radionuclide uptake; large, small, or no kidneys or abnormal differential function. Renal scarring was defined as the presence of decreased radionuclide uptake associated with loss of the uniform outline or the presence of cortical thinning with decreased volume (11). The classification of vesicoureteral reflux (VUR) in VCUG reports (grade I-V) was performed according to the international reflux study in children (12). The interpretations of DMSA scintigraphy and VCUG findings were performed by a single experienced nuclear medicine consultant and a single pediatric radiologist, respectively, which were blinded to the patients' clinical and laboratory characteristics. We divided the study cases into two groups based on presence of previous UTI to assess its effect on DMSA scan results. To examine the accuracy of abnormal DMSA scintigraphic results (consistent with acute pyelonephritis) in predicting VUR, sensitivity, specificity, positive predictive value (PPV), negative predictive value (NPV), and negative and positive likelihood ratio were also calculated. We used chi-square test or Fisher's exact, Mann-Whitney test for qualitative and $\mathrm{T}$ test for quantitative variables. $P$ values of 0.05 or less were considered statistically significant. All the statistical analyses were performed using SPSS version 16.0 (SPSS Inc., Chicago, IL, USA). 


\section{Results}

We retrospectively identified 208 children who presented with community acquired UTI, including 38 boys (18.3\%) and 170 girls. 23\% had a positive history of UTI but $77 \%$ gave no history of any previous UTI. Age range was 2-120 months ( $28 \pm 26$ months) and weight range was 3.247 Kilograms ( $12 \pm 6.4 \mathrm{Kgs})$.

The Age and Weight and the laboratory parameters of the patients in PreUTI (history of previous UTI) positive and Negative groups are shown in Table 1. Erythrocyte sedimentation rate(ESR) was more than $60 \mathrm{~mm} / \mathrm{hr}$ in $36.6 \%$ of the patients. Urinary RBC and WBC counts were reported 'many' in $8.6 \%$ and $30.7 \%$ of the children, respectively.

40 of 208 patients did not undergo DMSA for various reasons including refusal by their parents, rural location, and low socioeconomic status, but age and weight variables were not different statistically between two groups. Therefore, 168 children underwent DMSA within 2 days after the diagnosis of a febrile UTI and thus 336 renal units were included into the study (each pair of kidney is equivalent to two renal units). Abnormal DMSA scintigraphic results, suggestive of acute pyelonephritis, were found for 286 of 336 renal units. There were no significant differences in sex ratio, positive blood culture, positive CRP, detected hematuria, between the children with abnormal and normal DMSA findings. However, previous UTI was more prevalent in the former group $(P=0.01)$.

VCUG was performed in 290 renal units one month after the diagnosis and showed evidence of VUR in 49 (16.9\%). VUR was detected in 5 (14.0\%) of 36 renal units with normal DMSA results and 44 (17.3\%) of 254 renal units with abnormal DMSA results, in which high grade VUR (grade III-V) was detected in 18 (7.1\%) of the abnormal DMSA group and in 1(2.8\%) of the normal DMSA group $(P=0.56)$. In the group with previous UIT $(n=68)$, the sensitivity and specificity of abnormalities on DMSA renal scan to detect the presence of VUR (grade III - V) on VCUG were
100\% and 6\%. The PPV and NPV were $1 \%$ and $100 \%$, The LRpositive and negative for summarizing the utility of DMSA for ruling out the VUR (grade III - V) on VCUG was 1.06 and 0, respectively. In the group without evidence of previous UIT, the sensitivity and specificity of abnormalities on DMSA renal scan to detect the presence of VUR (grade III - V) on VCUG were $93 \%$ and $15 \%$. The PPV and NPV were $8 \%$ and $97 \%$, respectively. The LR positive and negative values for summarizing the utility of DMSA for ruling out the VUR (grade III - V) on VCUG were 1.09 and 0.46 respectively (Table 2).

\section{Discussion}

In this study, we evaluated whether early DMSA scintigraphic results ruled out the presence of high grade dilating VUR in children with symptomatic, communityacquired UTIs. In fact, we assessed the predictive power of DMSA compared to VCUG to predict VUR. In our research, about $85 \%$ of the children with UTIs had evidence of pyelonephritis on renal scanning with DMSA, which was a little higher than the rate in previous reports (13-15).

In our study, $16.9 \%$ of children with UTIs had VUR on VCUG, which was lower than the rate in previous reports (15). Of the renal units showing reflux, 7.1\% had grade III to $\mathrm{V}$ reflux which was considerably lower than what was reported in the Tseng et al. study (4). The NPV of pyelonephritis on DMSA renal scans to detect the presence of high grade VUR on VCUG in both children with or without history of UTIs was high (100\% and 97\%, respectively), which was in concordance with the previous retrospective study by Hansson (7), but was higher than what was reported by Hoberman (14). In addition, high sensitivity, high NPV, and very little negative LR of pyelonephritis on DMSA renal scan to detect the presence of VUR on VCUG were found in both groups $(7,14)$. As shown in this study, DMSA scintigraphy is a high sensitive test and is considered as the gold standard for diagnosis of renal damage

\begin{tabular}{lllll}
\hline \multicolumn{1}{l}{ Table 1. Baseline Variables in PreUTI Negative and Positive Cases } & & \\
\hline & PreUTI Positive $(\mathbf{n = 4 8})$ & PreUTI Negative $(\mathbf{n = 1 6 0})$ & $\boldsymbol{P}$-value & Confidence Interval 95\% \\
\hline UA WBC & $24.3 \pm 13$ & $24 \pm 15$ & NS & $-3.5-3.9$ \\
UA RBC & $5.4 \pm 8$ & $4.3 \pm 7$ & NS & $-0.8-3$ \\
ESR & $46.2 \pm 23$ & $43.8 \pm 30$ & NS & $-6.1-10.7$ \\
Age, mo & $39 \pm 32$ & $26 \pm 24$ & $<0.01$ & $6.3-20.2$ \\
Weight & $13.1 \pm 6.2$ & $11.9 \pm 7$ & NS & $-0.3-3$ \\
WBC & $12239 \pm 3600$ & $12832 \pm 5600$ & NS & $-20.7-831$ \\
HCT & $34.2 \pm 1.8$ & $32.2 \pm 5$ & 0.004 & $0.6-3$ \\
\hline
\end{tabular}

Abbreviations: PreUTI, a history of previous urinary tract infection; NS, non-significant

Table 2. Sensitivity and Specificity and PPV and NPV and Positive and Negative LR of Abnormality on DMSA renal scan to detect the presence of VUR (grade III - V) on VCUG in Total patients and children with UTI with or without history of previous UTI

\begin{tabular}{|c|c|c|c|c|c|c|}
\hline History of Previous UTI & Sensitivity, $\%$ & Specificity, \% & PPV, \% & NPV, $\%$ & Positive LR & Negative LR \\
\hline Total Patients & 94 & 12 & 7 & 97 & 1.06 & 0.5 \\
\hline Patients without Previous UTI $(n=68)$ & 93 & 15 & 8 & 97 & 1.09 & 0.49 \\
\hline Patients with Previous UTI $(\mathbf{n}=\mathbf{2 2 2})$ & 100 & 6 & 1 & 100 & 1.06 & 0 \\
\hline
\end{tabular}

Abbreviations: DMSA, dimercaptosuccinic acid; PPV, Positive Predictive Value; NPV, Negative Predictive value; VCUG, voiding cystourethrography; VUR,vesicoureteral reflux; UTI, urinary tract infection 
$(16,17)$. We found a high sensitivity of $94 \%$, a high negative predictive value of $97 \%$ and a low negative likelihood ratio ( 0.5 totally and 0 in children with previous history of UTI) of acute DMSA abnormality to detect VUR $\geq$ grade 3 , these results lower the risk of dilating VUR in children with normal DMSA scan findings, the difference among VUR grades was not statistically significant $(P=0.56)$ but only one child with normal DMSA values had VUR $\geq$ grade 3. This means that a negative DMSA renal scanning result decreases the probability of finding high grade VUR on VCUG, and it is a strong predictor of the absence of highgrade VUR in young children admitted with UTI. In our study, the reported PPV was very lower than what was reported in previous reported studies, which shows that we had cases with abnormal DMSA results without high grade VUR, but its high NPV minimizes the risk of missing the presence of VUR in children with pyelonephritis.

In fact, our finding suggests that in children with a previous history of UTI ( $0.23 \%$ of total cases), a negative result on a DMSA renal scan can be used to replace VCUG to rule out the possibility of high grade VUR which decreases the number of VCUG performed for UTI, and is important because of the drawbacks of this procedure, including unpleasantness to the child, possible risk of secondary infection, radiation exposure, and common refusal by parents to have their child tested. Our study reveals that this screening procedure is effective to detect high grade VUR in children with UTIs.

This study limitation was its retrospective pattern so we could not follow the patient results in future. The study was conducted in only one hospital but Mofid children hospital is a referral center. The missed cases were unavoidable because Iranian people do not desire to perform VCUG as an invasive procedure.

In children especially with a previous history of UTI ( $0.23 \%$ of total cases), VCUG can be omitted safely after a normal DMSA scan result, and at least in parents who refuse to perform VCUG, having a normal DMSA scan findings can be a reliable predictor of absences of VUR $\geq$ grade III, in total cases and children with no previous history of UTI high sensitivity and NPV of DMSA makes it a reliable test and low specificity and PPV cannot decrease its value as a screening test.

\section{Acknowledgments}

We kindly appreciate Pediatric Nephrology Ward and Laboratory of Mofid Hospital Personnel for their cooperation in Data assemblance and invaluable assistance of Dr Yaseri and Dr Ahmad Reza Shamshiri in statistical analysis.

\section{Authors' Contribution}

The work presented here was carried out in collaboration between all authors. Data collection was carried out by F.Shirvani and data analysis was done under supervision of Professor Sharifian and Mohkam.
Data collection and Statistical Analysis operations were Paid by Pediatric Infections Research Center.

\section{Funding/Support}

This study was under funding support of Pediatric Infections Research Center.

\section{References}

1. Twaij M. Urinary tract infection in children: a review of its pathogenesis and risk factors. J R Soc Promot Health. 2000;120(4):220-6.

2. Bachur R, Harper MB. Reliability of the urinalysis for predicting urinary tract infections in young febrile children. Arch Pediatr Adolesc Med. 2001;155(1):60-5.

3. Ajdinovic B, Jaukovic L, Krstic Z, Dopuda M. Impact of micturating cystourethrography and DMSA renal scintigraphy on the investigation scheme in children with urinary tract infection. Ann Nucl Med. 2008;22(8):661-5.

4. Tseng MH, Lin WJ, Lo WT, Wang SR, Chu ML, Wang CC. Does a normal DMSA obviate the performance of voiding cystourethrography in evaluation of young children after their first urinary tract infection? J Pediatr. 2007;150(1):96-9.

5. Lin CH, Yang LY, Wamg HH, Chang JW, Shen MC, Tang RB. Evaluation of imaging studies for vesicoureteral reflux in infants with first urinary tract infection. Acta Pediatr Taiwan. 2007;48(2):68-72.

6. Lee MD, Lin CC, Huang FY, Tsai TC, Huang CT, Tsai JD. Screening young children with a first febrile urinary tract infection for high-grade vesicoureteral reflux with renal ultrasound scanning and technetium-99m-labeled dimercaptosuccinic acid scanning. J Pediatr. 2009;154(6):797-802.

7. Hansson S, Dhamey M, Sigstrom O, Sixt R, Stokland E, Wennerstrom M, et al. Dimercapto-succinic acid scintigraphy instead of voiding cystourethrography for infants with urinary tract infection.J Urol. 2004;172(3):1071-3; discussion 3-4.

8. Swerkersson S, Andreasson AC, Jodal U, Sixt R, Stockland E, Hansson $S$. The insignificance of low-grade vesicoureteral reflux Pediatr Nephrol. 2006;10:1511.

9. Preda I, Jodal U, Sixt R, Stokland E, Hansson S. Normal dimercaptosuccinic acid scintigraphy makes voiding cystourethrography unnecessary after urinary tract infection. J Pediatr. 2007;151(6):581-4, 4 e1.

10. Siomou E, Giapros V, Fotopoulos A, Aasioti M, Papadopoulou F, Serbis A, et al. Implications of 99mTc-DMSA scintigraphy performed during urinary tract infection in neonates. Pediatrics. 2009;124(3):881-7.

11. Patel K, Charron M, Hoberman A, Brown ML, Rogers KD. Intraand interobserver variability in interpretation of DMSA scans using a set of standardized criteria. Pediatr Radiol. 1993;23(7):506-9.

12. Lebowitz RL, Olbing H, Parkkulainen KV, Smellie JM, TamminenMobius TE. International system of radiographic grading of vesicoureteric reflux. International Reflux Study in Children. Pediatr Radiol. 1985;15(2):105-9.

13. Yen CW, Chen DH. Urinary tract infection in children.J Microbiol Immunol Infect. 1999;32(3):199-205.

14. Hoberman A, Charron M, Hickey RW, Baskin M, Kearney DH, Wald ER. Imaging studies after a first febrile urinary tract infection in young children. N Engl J Med. 2003;348(3):195-202.

15. Lin KY, Chiu NT, Chen MJ, Lai CH, Huang JJ, Wang YT, et al. Acute pyelonephritis and sequelae of renal scar in pediatric first febrile urinary tract infection. Pediatr Nephrol. 2003;18(4):362-5.

16. Rushton HG, Majd M, Chandra R, Yim D. Evaluation of 99mtechnetium-dimercapto-succinic acid renal scans in experimental acute pyelonephritis in piglets. J Urol. 1988;140(5 Pt 2):1169-74.

17. Majd M, Nussbaum Blask AR, Markle BM, Shalaby-Rana E, Pohl HG Park JS, et al. Acute pyelonephritis: comparison of diagnosis with 99mTc-DMSA, SPECT, spiral CT, MR imaging, and power Doppler US in an experimental pig model. Radiology. 2001;218(1):101-8.

\section{Financial Disclosure}

